\title{
Editorial
}

\section{Real-Life Applications of Fuzzy Logic}

\author{
Harpreet Singh, ${ }^{1}$ Madan M. Gupta, ${ }^{2}$ Thomas Meitzler, ${ }^{3}$ Zeng-Guang Hou, ${ }^{4}$ \\ Kum Kum Garg, ${ }^{5}$ Ashu M. G. Solo, ${ }^{6}$ and Lotfi A. Zadeh ${ }^{7}$ \\ ${ }^{1}$ Department of Electrical and Computer Engineering, Wayne State University, Detroit, MI 48202, USA \\ ${ }^{2}$ Intelligent Systems Research Laboratory, College of Engineering, University of Saskatchewan, Saskatoon, SK, Canada S7N 5A9 \\ ${ }^{3}$ Electrified Armor Lab, RDECOM TARDEC, 6501 E. 11 Mile Road, RDTA-RS, MS 263, Warren, MI 48397-5000, USA \\ ${ }^{4}$ Key Laboratory of Complex Systems and Intelligence Science, Institute of Automation, Chinese Academy of Sciences, \\ P.O. Box 2728, Beijing 100190, China \\ ${ }^{5}$ Manipal University Jaipur, Jaipur 303007, India \\ ${ }^{6}$ Maverick Technologies America Inc., Suite 808, 1220 North Market Street, Wilmington, DE 19801, USA \\ ${ }^{7}$ Department of Electrical Engineering and Computer Sciences, University of California Berkeley, CA 94720-1776, USA
}

Correspondence should be addressed to Harpreet Singh; hsingh@eng.wayne.edu

Received 18 April 2013; Accepted 18 April 2013

Copyright (C) 2013 Harpreet Singh et al. This is an open access article distributed under the Creative Commons Attribution License, which permits unrestricted use, distribution, and reproduction in any medium, provided the original work is properly cited.

This special issue is dedicated to Professor Lotfi A. Zadeh, the father of fuzzy logic

The theory of fuzzy logic is based on the notion of relative graded membership, as inspired by the processes of human perception and cognition. Lotfi A. Zadeh published his first famous research paper on fuzzy sets in 1965. Fuzzy logic can deal with information arising from computational perception and cognition, that is, uncertain, imprecise, vague, partially true, or without sharp boundaries. Fuzzy logic allows for the inclusion of vague human assessments in computing problems. Also, it provides an effective means for conflict resolution of multiple criteria and better assessment of options. New computing methods based on fuzzy logic can be used in the development of intelligent systems for decision making, identification, pattern recognition, optimization, and control.

Fuzzy logic is extremely useful for many people involved in research and development including engineers (electrical, mechanical, civil, chemical, aerospace, agricultural, biomedical, computer, environmental, geological, industrial, and mechatronics), mathematicians, computer software developers and researchers, natural scientists (biology, chemistry, earth science, and physics), medical researchers, social scientists (economics, management, political science, and psychology), public policy analysts, business analysts, and jurists.
Indeed, the applications of fuzzy logic, once thought to be an obscure mathematical curiosity, can be found in many engineering and scientific works. Fuzzy logic has been used in numerous applications such as facial pattern recognition, air conditioners, washing machines, vacuum cleaners, antiskid braking systems, transmission systems, control of subway systems and unmanned helicopters, knowledge-based systems for multiobjective optimization of power systems, weather forecasting systems, models for new product pricing or project risk assessment, medical diagnosis and treatment plans, and stock trading. Fuzzy logic has been successfully used in numerous fields such as control systems engineering, image processing, power engineering, industrial automation, robotics, consumer electronics, and optimization. This branch of mathematics has instilled new life into scientific fields that have been dormant for a long time.

Thousands of researchers are working with fuzzy logic and producing patents and research papers. According to Zadeh's report on the impact of fuzzy logic as of March 4, 2013 , there are 26 research journals on theory or applications of fuzzy logic, there are 89,365 publications on theory or applications of fuzzy logic in the INSPEC database, there are 
22,657 publications on theory or applications of fuzzy logic in the MathSciNet database, there are 16,898 patent applications and patents issued related to fuzzy logic in the USA, and there are 7149 patent applications and patents issued related to fuzzy logic in Japan. The number of research contributions is growing daily and is growing at an increasing rate. Zadeh started the Berkeley Initiative in Soft Computing (BISC), a famous research laboratory at University of California, Berkeley, to advance theory and applications of fuzzy logic and soft computing.

The objective of this special issue is to explore the advances of fuzzy logic in a large number of real-life applications and commercial products in a variety of fields. Although fuzzy logic has applications in a number of different areas, it is not yet known to people unfamiliar with intelligent systems how it can be applied in different products that are currently available in the market. For many people, the engineering and scientific meaning of the word fuzzy is still fuzzy. It is important that these people understand where and how fuzzy logic can be used.

In "Detection and elimination of a potential fire in engine and battery compartments of hybrid electric vehicles" by M. S. Dattathreya et al, the authors present a novel fuzzy deterministic noncontroller type (FDNCT) system and an FDNCT inference algorithm (FIA). The FDNCT is used in an intelligent system for detecting and eliminating potential fires in the engine and battery compartments of a hybrid electric vehicle. They also present the simulation results of the comparison between the FIA and singleton inference algorithms for detecting potential fires and determining the actions for eliminating them.

In "Comparison of detection and classification algorithms using boolean and fuzzy techniques" by R. Dixit and H. Singh, the authors compare various logic analysis methods and present results for a hypothetical target classification scenario. They show how preprocessing can reasonably preserve result confidence and compare the results between Boolean, multiquantization Boolean, and fuzzy techniques.

In "BDD, BNN, and FPGA on fuzzy techniques for rapid system analysis" by R. Dixit and H. Singh, the authors look at techniques to simplify data analysis of large multivariate military sensor systems.

In "A fuzzy preprocessing module for optimizing the access network selection in wireless networks" by F. Kaleem et al, the authors present the design and implementation of a fuzzy multicriteria scheme for vertical handoff necessity estimation. Their method determines the proper time for vertical handoff while considering the continuity and quality of the currently utilized service and end-user satisfaction.

In "A soft computing approach to crack detection and impact source identification with field-programmable gate array implementation" by A. M. Dixit and H. Singh, the authors present a fuzzy inference system to automate crack detection and impact source identification (CDISI) and present their work on a microchip for automated CDISI.

In "Analysis of adaptive fuzzy technique for multiple crack diagnosis of faulty beam using vibration signatures" by A. K. Dash, the author proposes a method for multicrack detection of structure using a fuzzy Gaussian technique.
In "Effect of road traffic noise pollution on human work efficiency in government offices, private organizations, and commercial business centres in agartala city using fuzzy expert system: a case study" by D. Pal and D. Bhattacharya, the authors examine the reduction in human work efficiency due to growing road traffic noise pollution. Using fuzzy logic, they monitor and model disturbances from vehicular road traffic and the effect on personal work performance.

In "A Hybrid approach to failure analysis using stochastic petri nets and ranking generalized fuzzy numbers" by A. D. Torshizi and J. Parvizian, the authors present an innovative failure analysis approach that combines the flexibility of fuzzy logic with the structural properties of stochastic Petri nets. This algorithm has a diverse range of industrial applications.

In "Excluded-mean-variance neural decision analyzer for qualitative group decision making" by K.-Y. Song et al, the authors introduce an innovative mean-variance neural approach for group decision making in uncertain situations. The authors provide a case study with the excluded-meanvariance approach that shows that this approach can improve the effectiveness of qualitative decision making by providing the decision maker with a new cognitive tool to assist in the reasoning process.

In "Warren, McCain, and Obama needed fuzzy sets at presidential forum" by A. M. G. Solo, the author shows how the moderator and presidential candidates in a presidential forum needed fuzzy logic to properly ask and answer a debate question. The author shows how an understanding of fuzzy logic is needed to properly ask and answer queries about defining imprecise linguistic terms. Then A. M. G. Solo distinguishes between qualitative definitions and quantitative definitions of imprecise linguistic terms and between crisp quantitative definitions and fuzzy quantitative definitions of imprecise linguistic terms.

In "A fuzzy rule-based expert system for evaluating intellectual capital" by M. H. F. Zarandi et al, the authors describe their fuzzy expert system for evaluating intellectual capital. This assists managers in understanding and evaluating the level of each asset created through intellectual activities.

There were 20 research papers submitted. Only 11 research papers were accepted. Therefore, 55\% of submitted research papers were accepted. Research papers were accepted from 22 researchers at 13 universities and research institutions in the USA, Canada, India, Japan, and Iran.

This special issue describes many important research advancements in real-life applications of fuzzy logic. Also, it creates awareness of real-life applications of fuzzy logic and thereby encourages others to do research and development in more real-life applications of fuzzy logic. There are numerous other applications of fuzzy logic that have to be researched and developed.

Harpreet Singh Madan M. Gupta Thomas Meitzler Zeng-Guang Hou Kum Kum Garg Ashu M. G. Solo 


\section{Foreword by Lotfi A. Zadeh, Father of Fuzzy Logic}

I am deeply appreciative of the dedication to me of this special issue, of the journal of Advances in Fuzzy Systems. Additionally, I appreciate very much being asked by the editors to contribute a brief foreword. For me, the foreword is an opportunity to offer a comment on the theme of the special issue.

First, a bit of history, my 1965 paper on fuzzy sets was motivated by my feeling that the then existing theories provided no means of dealing with a pervasive aspect of realityunsharpness (fuzziness) of class boundaries. Without such means, realistic models of human-centered and biological systems are hard to construct. My expectation was that fuzzy set theory would be welcomed by the scientific communities in these and related fields. Contrary to my expectation, in these fields, fuzzy set theory was met with skepticism and, in some instances, with hostility. What I did not anticipate was that, for many years after the debut of fuzzy set theory, its main applications would be in the realms of engineering systems and consumer products.

The first significant real-life applications of fuzzy set theory and fuzzy logic began to appear in the late seventies and early eighties. Among such applications were fuzzy logiccontrolled cement kilns and production of steel. The first consumer product was Matsushita's shower head, 1986. Soon, many others followed, among them home appliances, photographic equipment, and automobile transmissions. A major real-life application was Sendai's fuzzy logic control system which began to operate in 1987 and was and is a striking success. In the realm of medical instrumentation, a notable real-life application is Omron's fuzzy- logic-based and widely used blood pressure meter.

The past two decades have witnessed a significant change in the nature of applications of fuzzy logic. Nonengineering applications have grown in number, visibility, and importance. Among such applications are applications in medicine, social sciences, policy sciences, fraud detection systems, assessment of credit-worthiness systems, and economics. Particularly worthy of note is the path-breaking work of Professor Rafik Aliev on application of fuzzy logic to decision making in the realm of economics. Once his work is understood, it is certain to have a major impact on economic theories.

Underlying real-life applications of fuzzy logic is a key idea. Almost all real-life applications of fuzzy logic involve the use of linguistic variables. A linguistic variable is a variable whose values are words rather than numbers. The concept of a linguistic variable was introduced in my 1973 paper. In science, there is a deep-seated tradition of according much more respect for numbers than for words. In fact, scientific progress is commonly equated to progression from the use of words to the use of numbers. My counter traditional suggestion to use words in place of numbers made me an object of severe criticism and derision from prominent members of the scientific community. The point which I was trying to make was not understood.

Underlying the concept of a linguistic variable is a fact which is widely unrecognized-a fact which relates to the concept of precision. Precision has two distinct meanings - precision in value and precision in meaning. The first meaning is traditional. The second meaning is not. The second meaning is rooted in fuzzy logic. Example. Consider the proposition, p: Robert is young. So far as Robert's age is concerned, $\mathrm{p}$ is imprecise in value, but so far as meaning is concerned, $\mathrm{p}$ is precise in meaning if tall is interpreted as a fuzzy set with a specified membership function. More concretely, when in fuzzy logic a word represents the value of a variable, the word is precisiated by treating it as a specified fuzzy set. This is the key idea which underlies the concept of a linguistic variable-an idea which opens the door to exploitation of tolerance for imprecision. Precision carries a cost. When there is some tolerance for imprecision, the use of words serves to reduce cost. Equally importantly, the use of words serves to construct better models of reality. This is what my prominent critics did not appreciate. There is a lesson to be learned.

In conclusion, a word about the methodology of computing with words (CWW). CWW is rooted in the concept of a linguistic variable. CWW opens the door to construction of mathematical solutions of computational problems which are stated in natural language. In coming years, CWW is likely to play an increasingly important role in origination and development of real-life applications of fuzzy logic.

Lotfi A. Zadeh 

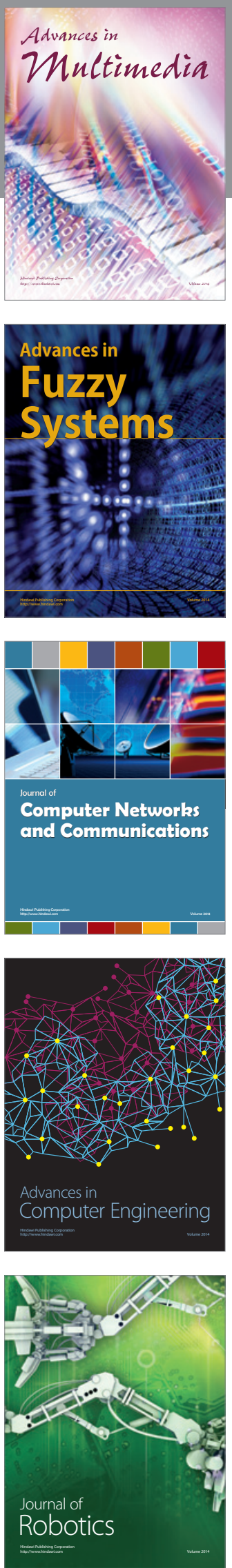

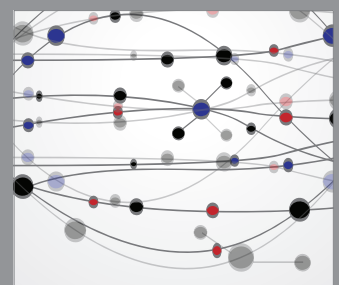

The Scientific World Journal
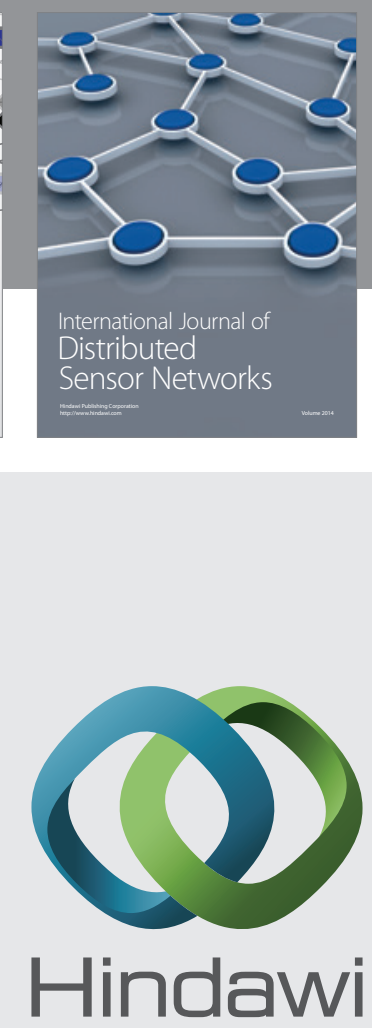

Submit your manuscripts at

http://www.hindawi.com
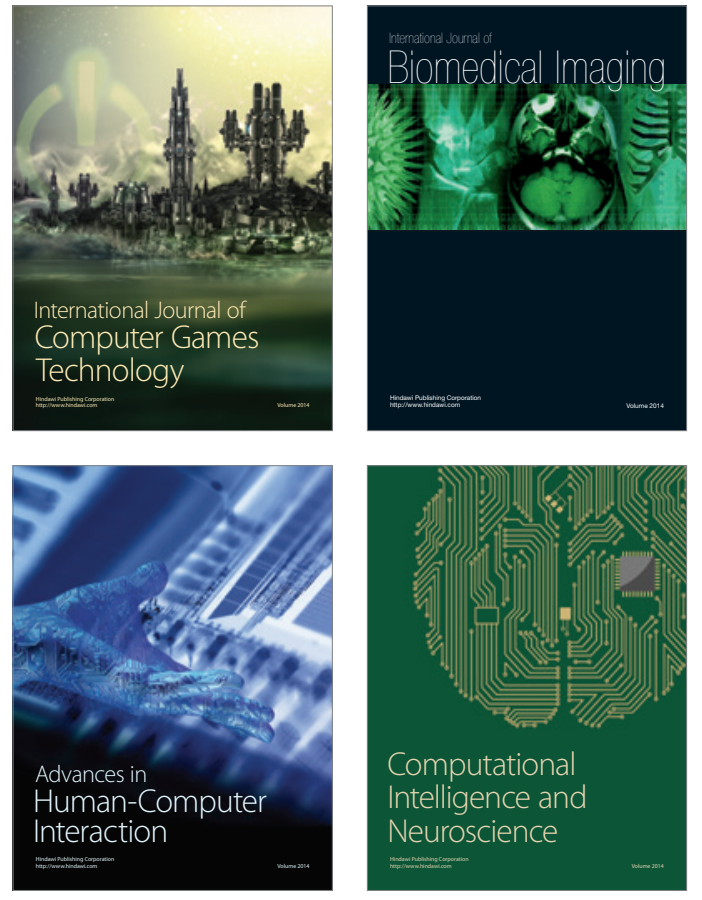
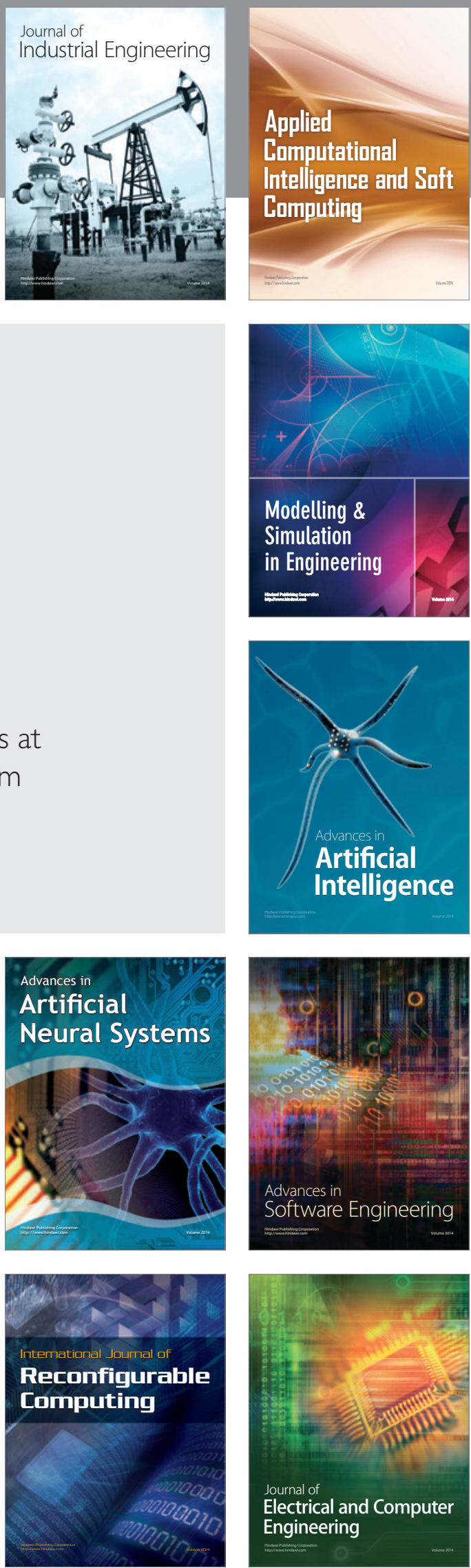